\title{
A Cohen Type Inequality for Fourier Expansions of Orthogonal Polynomials with a Nondiscrete Jacobi-Sobolev Inner Product
}

\author{
Bujar Xh. Fejzullahu' ${ }^{1}$ and Francisco Marcellán ${ }^{2}$ \\ ${ }^{1}$ Department of Mathematics, Faculty of Mathematics and Natural Sciences, University of Prishtina, \\ Mother Teresa 5, Prishtinë 10000, Kosovo \\ ${ }^{2}$ Departamento de Matemáticas, Escuela Politécnica Superior, Universidad Carlos III de Madrid, \\ Avenida de la Universidad 30, 28911 Leganés, Spain
}

Correspondence should be addressed to Francisco Marcellán, pacomarc@ing.uc3m.es

Received 5 May 2010; Accepted 24 August 2010

Academic Editor: Józef Banaś

Copyright (C) 2010 B. Xh. Fejzullahu and F. Marcellán. This is an open access article distributed under the Creative Commons Attribution License, which permits unrestricted use, distribution, and reproduction in any medium, provided the original work is properly cited.

Let $\left\{Q_{n}^{(\alpha, \beta)}(x)\right\}_{n \geq 0}$ denote the sequence of polynomials orthogonal with respect to the non-discrete Sobolev inner product $\langle f, g\rangle=\int_{-1}^{1} f(x) g(x) d \mu_{\alpha, \beta}(x)+\lambda \int_{-1}^{1} f^{\prime}(x) g^{\prime}(x) d \mu_{\alpha+1, \beta}(x)$, where $\lambda>0$ and $d \mu_{\alpha, \beta}(x)=(1-x)^{\alpha}(1+x)^{\beta} d x$ with $\alpha>-1, \beta>-1$. In this paper, we prove a Cohen type inequality for the Fourier expansion in terms of the orthogonal polynomials $\left\{Q_{n}^{(\alpha, \beta)}(x)\right\}_{n}$. Necessary conditions for the norm convergence of such a Fourier expansion are given. Finally, the failure of almost everywhere convergence of the Fourier expansion of a function in terms of the orthogonal polynomials associated with the above Sobolev inner product is proved.

\section{Introduction}

Let $d \mu_{\alpha, \beta}(x)=(1-x)^{\alpha}(1+x)^{\beta} d x$ with $\alpha, \beta>-1$ be the Jacobi measure supported on the interval $[-1,1]$. We say that $f \in L^{p}\left(d \mu_{\alpha, \beta}\right)$ if $f$ is measurable on $[-1,1]$ and $\|f\|_{L^{p}\left(d \mu_{\alpha, \beta}\right)}<\infty$, where

$$
\|f\|_{L^{p}\left(d \mu_{\alpha, \beta}\right)}= \begin{cases}\left(\int_{-1}^{1}|f(x)|^{p} d \mu_{\alpha, \beta}(x)\right)^{1 / p}, & \text { if } 1 \leq p<\infty, \\ \underset{-1<x<1}{\operatorname{essup}|f(x)|,} & \text { if } p=\infty .\end{cases}
$$


Let us introduce the Sobolev-type spaces (see, for instance, [1, Chapter III], in a more general framework) as follows:

$$
\begin{gathered}
S_{p}^{\alpha, \beta}=\left\{f:\|f\|_{S_{p}^{\alpha, \beta}}^{p}=\|f\|_{L^{p}\left(d \mu_{\alpha, \beta}\right)}^{p}+\lambda\left\|f^{\prime}\right\|_{L^{p}\left(d \mu_{\alpha+1, \beta}\right)}^{p}<\infty\right\}, \quad 1 \leq p<\infty, \\
S_{\infty}^{\alpha, \beta}=\left\{f:\|f\|_{S_{\infty}^{\alpha, \beta}}=\max \left\{\|f\|_{L^{\infty}\left(d \mu_{\alpha, \beta}\right)},\left\|f^{\prime}\right\|_{L^{\infty}\left(d \mu_{\alpha+1, \beta}\right)}\right\}<\infty\right\},
\end{gathered}
$$

where $\lambda>0$, as well as the linear space $\left[S_{p}^{\alpha, \beta}\right]$ of all bounded linear operators $T: S_{p}^{\alpha, \beta} \rightarrow S_{p}^{\alpha, \beta}$, with the usual operator norm

$$
\|T\|_{\left[S_{p}^{\alpha, \beta}\right]}=\sup _{0 \neq f \in S_{p}^{\alpha, \beta}} \frac{\|T(f)\|_{S_{p}^{\alpha, \beta}}}{\|f\|_{S_{p}^{\alpha, \beta}}} .
$$

Let $f$ and $g$ be in $S_{2}^{\alpha, \beta}$. Let us consider the following Sobolev-type inner product:

$$
\langle f, g\rangle=\int_{-1}^{1} f(x) g(x) d \mu_{\alpha, \beta}(x)+\lambda \int_{-1}^{1} f^{\prime}(x) g^{\prime}(x) d \mu_{\alpha+1, \beta}(x),
$$

where $\lambda>0$. Let $\left\{Q_{n}^{(\alpha, \beta)}(x)\right\}_{n=0}^{\infty}$ denote the sequence of polynomials orthogonal with respect to (1.4), normalized by the condition that $Q_{n}^{(\alpha, \beta)}$ has the same leading coefficient as the following classical Jacobi polynomial:

$$
P_{n}^{(\alpha, \beta-1)}(x)=\frac{1}{2^{n}}\left(\begin{array}{c}
2 n+\alpha+\beta-1 \\
n
\end{array}\right) x^{n}+\text { lower degree terms. }
$$

We call them the Jacobi-Sobolev orthogonal polynomials.

The measures $\mu_{\alpha, \beta}$ and $\mu_{\alpha+1, \beta}$ constitute a particular case of the so-called coherent pairs of measures studied in [2]. In [3] (see also [4]), the authors established the asymptotics of the zeros of such Jacobi-Sobolev polynomials.

The aim of our contribution is to obtain a lower bound for the norm of the partial sums of the Fourier expansion in terms of Jacobi-Sobolev polynomials, the well-known Cohen type inequality in the framework of Approximation Theory. A Cohen type inequality has been established in other contexts, for example, on compact groups or for classical orthogonal expansions. See [5-10] and references therein.

Throughout the paper, positive constants are denoted by $c, c_{1}, \ldots$ and they may vary at every occurrence. The notation $u_{n} \cong v_{n}$ means that the sequence $u_{n} / v_{n}$ converges to 1 and $u_{n} \sim v_{n}$ means $c_{1} u_{n} \leq v_{n} \leq c_{2} u_{n}$ for sufficiently large $n$, where $c_{1}$ and $c_{2}$ are positive real numbers.

The structure of the paper is as follows. In Section 2, we introduce the basic background about Jacobi polynomials to be used in the paper. In particular, we focus our attention in some estimates and the strong asymptotics on $[-1,1]$ for such polynomials as well as the Mehler-Heine formula. In Section 3, we analyze the polynomials orthogonal with respect to the inner product (1.4). Their representation in terms of Jacobi polynomials yields 
estimates, inner strong asymptotics, and a Mehler-Heine type formula. Some estimates of the weighted $p$ Sobolev norm of these polynomials will be needed in the sequel and we show them in Proposition 3.12. In Section 4, a Cohen-type inequality, associated with the Fourier expansions in terms of the Jacobi-Sobolev orthogonal polynomials, is deduced. In Section 5, we focus our attention in the norm convergence of the above Fourier expansions. Finally, Section 6 is devoted to the analysis of the divergence almost everywhere of such expansions.

\section{Jacobi Polynomials}

For $\alpha, \beta>-1$, we denote by $\left\{P_{n}^{(\alpha, \beta)}(x)\right\}_{n=0}^{\infty}$ the sequence of Jacobi polynomials which are orthogonal on $[-1,1]$ with respect to the measure $d \mu_{\alpha, \beta}$. They are normalized in such a way that $P_{n}^{(\alpha, \beta)}(1)=\left(\begin{array}{c}n+\alpha \\ n\end{array}\right)$. We denote the $n$th monic Jacobi polynomial by

$$
\widehat{P}_{n}^{(\alpha, \beta)}(x)=\left(h_{n}^{\alpha, \beta}\right)^{-1} P_{n}^{(\alpha, \beta)}(x)
$$

where (see [11, formula (22.3.1)])

$$
h_{n}^{\alpha, \beta}=\frac{1}{2^{n}}\left(\begin{array}{c}
2 n+\alpha+\beta \\
n
\end{array}\right) \text {. }
$$

Now, we list some basic properties of Jacobi polynomials which will be used in the sequel. The following integral formula for Jacobi polynomials holds (see (2.1) and [11, formula (22.2.1)]):

$$
\begin{aligned}
\int_{-1}^{1}[ & \left.\widehat{P}_{n}^{(\alpha, \beta)}(x)\right]^{2} d \mu_{\alpha, \beta}(x) \\
& =2^{2 n+\alpha+\beta+1} \frac{\Gamma(n+1) \Gamma(n+\alpha+1) \Gamma(n+\beta+1) \Gamma(n+\alpha+\beta+1)}{\Gamma(2 n+\alpha+\beta+1) \Gamma(2 n+\alpha+\beta+2)} .
\end{aligned}
$$
follows:

They satisfy a connection formula (see [11, formula (22.7.19)], [3, formula (2.5)]) as

$$
\widehat{P}_{n}^{(\alpha, \beta-1)}(x)=\widehat{P}_{n}^{(\alpha, \beta)}(x)+a_{n-1}(\alpha, \beta) \widehat{P}_{n-1}^{(\alpha, \beta)}(x)
$$

where

$$
a_{n}(\alpha, \beta)=\frac{2(n+1)(n+\alpha+1)}{(2 n+\alpha+\beta+1)(2 n+\alpha+\beta+2)}, \quad n \geq 0,
$$

as well as the following relation for the derivatives (see [12, formula (4.21.7)]):

$$
\frac{d}{d x} P_{n}^{(\alpha, \beta-1)}(x)=\frac{n+\alpha+\beta}{2} P_{n-1}^{(\alpha+1, \beta)}(x)
$$


The following estimate for $P_{n}^{(\alpha, \beta)}$ holds (see [12, formula (7.32.6)], [13]):

$$
\left|P_{n}^{(\alpha, \beta)}(x)\right| \leq c n^{-1 / 2}(1-x)^{-\alpha / 2-1 / 4}(1+x)^{-\beta / 2-1 / 4},
$$

where $x \in(-1,1)$ and $\alpha, \beta \geq-1 / 2$.

The formula of Mehler-Heine for Jacobi orthogonal polynomials is (see [12, Theorem 8.1.1]) as follows:

$$
\lim _{n \rightarrow \infty} n^{-\alpha} P_{n}^{(\alpha, \beta)}\left(\cos \frac{z}{n}\right)=\left(\frac{z}{2}\right)^{-\alpha} J_{\alpha}(z)
$$

where $\alpha, \beta$ are real numbers, and $J_{\alpha}(z)$ is the Bessel function. This formula holds locally uniformly, that is, on every compact subset of the complex plane.

The inner strong asymptotics of $P_{n}^{(\alpha, \beta)}$, for $\theta \in[\epsilon, \pi-\epsilon]$ and $\epsilon>0$, are read as follows (see [12, Theorem 8.21.8]):

$$
P_{n}^{(\alpha, \beta)}(\cos \theta)=\pi^{-1 / 2} n^{-1 / 2}\left[\left(\sin \frac{\theta}{2}\right)^{-\alpha-1 / 2}\left(\cos \frac{\theta}{2}\right)^{-\beta-1 / 2} \cos (k \theta+\gamma)+O\left(n^{-1}\right)\right],
$$

where $k=n+(\alpha+\beta+1) / 2$, and $\gamma=-(\alpha+1 / 2) \pi / 2$.

For $\alpha, \beta, \mu>-1$ and $1 \leq q \leq \infty$ (see [12, page 391. Exercise 91], as well as [10, (2.2)])

$$
\left(\int_{0}^{1}(1-x)^{\mu}\left|P_{n}^{(\alpha, \beta)}(x)\right|^{p} d x\right)^{1 / p} \sim \begin{cases}n^{-1 / 2}, & \text { if } 2 \mu>p \alpha-2+\frac{p}{2} \\ n^{-1 / 2}(\log n)^{1 / p}, & \text { if } 2 \mu=p \alpha-2+\frac{p}{2} \\ n^{\alpha-(2 \mu+2) / p}, & \text { if } 2 \mu<p \alpha-2+\frac{p}{2}\end{cases}
$$

\section{Asymptotics of Jacobi-Sobolev Orthogonal Polynomials}

Let us denote by $\widehat{Q}_{n}^{(\alpha, \beta)}$ the monic Jacobi-Sobolev polynomial of degree $n$, that is, $\widehat{Q}_{n}^{(\alpha, \beta)}(x)=$ $\left(h_{n}^{\alpha, \beta-1}\right)^{-1} Q_{n}^{(\alpha, \beta)}(x)$. From $(2.4)$ and $[3$, formula $(2.7)]$ (see also $[4,14]$ in a more general framework), we have the following relation between the Jacobi-Sobolev and Jacobi monic orthogonal polynomials.

Proposition 3.1. For $\alpha, \beta>-1$,

$$
\widehat{P}_{n}^{(\alpha, \beta)}(x)+a_{n-1}(\alpha, \beta) \widehat{P}_{n-1}^{(\alpha, \beta)}(x)=\widehat{Q}_{n}^{(\alpha, \beta)}(x)+\widehat{d}_{n-1}(\lambda) \widehat{Q}_{n-1}^{(\alpha, \beta)}(x), \quad n \geq 1,
$$

where $a_{n-1}(\alpha, \beta)$ is given in (2.5) and

$$
\widehat{d}_{n}(\lambda)=a_{n}(\alpha, \beta) \frac{\left\|\widehat{P}_{n}^{(\alpha, \beta)}\right\|_{L^{2}\left(d \mu_{\alpha, \beta}\right)}^{2}}{\left\|\widehat{Q}_{n}^{(\alpha, \beta)}\right\|_{S_{2}^{\alpha}}^{2}}, \quad n \geq 0
$$


Proposition 3.2. One gets:

$$
\left\|\widehat{Q}_{n}^{(\alpha, \beta)}\right\|_{S_{2}^{\alpha, \beta}}^{2} \cong \lambda n^{2}\left\|\widehat{P}_{n-1}^{(\alpha+1, \beta)}\right\|_{L^{2}\left(d \mu_{\alpha+1, \beta}\right)}^{2}
$$

In particular, for $\widehat{d}_{n}(\lambda)$ defined in (3.2) one obtains

$$
\widehat{d}_{n}(\lambda) \cong \frac{1}{4 \lambda n^{2}}
$$

Proof. We apply the same argument as in the proof of Theorem 2 in [15]. Using the extremal property

$$
\left\|\widehat{P}_{n}^{(\alpha, \beta)}\right\|_{L^{2}\left(d \mu_{\alpha, \beta}\right)}^{2}=\inf \left\{\|P\|_{L^{2}\left(d \mu_{\alpha, \beta}\right)}^{2}: \operatorname{deg} P=n, P \text { monic }\right\}
$$

we get the following:

$$
\left\|\widehat{Q}_{n}^{(\alpha, \beta)}\right\|_{S_{2}^{\alpha, \beta}}^{2}=\left\|\widehat{Q}_{n}^{(\alpha, \beta)}\right\|_{L^{2}\left(d \mu_{\alpha, \beta}\right)}^{2}+\lambda\left\|{\widehat{Q_{n}^{\prime}}}^{(\alpha, \beta)}\right\|_{L^{2}\left(d \mu_{\alpha+1, \beta}\right)}^{2} \geq\left\|\widehat{P}_{n}^{(\alpha, \beta)}\right\|_{L^{2}\left(d \mu_{\alpha, \beta}\right)}^{2}+\lambda n^{2}\left\|\widehat{P}_{n-1}^{(\alpha+1, \beta)}\right\|_{L^{2}\left(d \mu_{\alpha+1, \beta}\right)}^{2}
$$

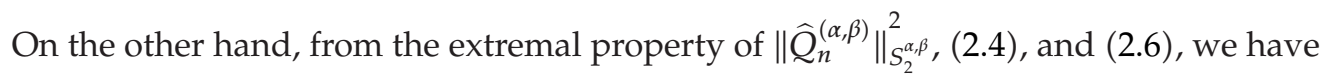

$$
\begin{aligned}
\left\|\widehat{Q}_{n}^{(\alpha, \beta)}\right\|_{S_{2}^{\alpha, \beta}}^{2} & \leq\left\|\widehat{P}_{n}^{(\alpha, \beta)}+a_{n-1}(\alpha, \beta) \widehat{P}_{n-1}^{(\alpha, \beta)}\right\|_{S_{2}^{\alpha, \beta}}^{2} \\
& =\left\|\widehat{P}_{n}^{(\alpha, \beta)}+a_{n-1}(\alpha, \beta) \widehat{P}_{n-1}^{(\alpha, \beta)}\right\|_{L^{2}\left(d \mu_{\alpha, \beta}\right)}^{2}+\lambda n^{2}\left\|\widehat{P}_{n-1}^{(\alpha+1, \beta)}\right\|_{L^{2}\left(d \mu_{\alpha+1, \beta}\right)}^{2} \\
& \leq\left\|\widehat{P}_{n}^{(\alpha, \beta)}\right\|_{L^{2}\left(d \mu_{\alpha, \beta}\right)}^{2}+\left(a_{n-1}(\alpha, \beta)\right)^{2}\left\|\widehat{P}_{n-1}^{(\alpha, \beta)}\right\|_{L^{2}\left(d \mu_{\alpha, \beta}\right)}^{2}+\lambda n^{2}\left\|\widehat{P}_{n-1}^{(\alpha+1, \beta)}\right\|_{L^{2}\left(d \mu_{\alpha+1, \beta}\right)}^{2} .
\end{aligned}
$$

Since by (2.3) and (2.5) we have $\left\|\widehat{P}_{n}^{(\alpha, \beta)}\right\|_{L^{2}\left(d \mu_{\alpha, \beta}\right)} \cong\left\|\widehat{P}_{n-1}^{(\alpha+1, \beta)}\right\|_{L^{2}\left(d \mu_{\alpha+1, \beta}\right)}$ and $a_{n}(\alpha, \beta) \cong 1 / 2$, then (3.6) and (3.7) yield (3.3).

As a straightforward consequence of Propositions 3.1 and 3.2, using (2.1) we deduce the following.

Corollary 3.3. For $\alpha, \beta>-1$,

$$
\frac{n+\alpha+\beta}{2 n+\alpha+\beta} P_{n}^{(\alpha, \beta)}(x)+\frac{n+\alpha}{2 n+\alpha+\beta} P_{n-1}^{(\alpha, \beta)}(x)=Q_{n}^{(\alpha, \beta)}(x)+d_{n-1}(\lambda) Q_{n-1}^{(\alpha, \beta)}(x)
$$


where $n \geq 1$ and

$$
d_{n}(\lambda)=\widehat{d}_{n}(\lambda) \frac{h_{n}^{\alpha, \beta-1}}{h_{n-1}^{\alpha, \beta-1}} \cong \frac{1}{2 \lambda n^{2}}
$$

Corollary 3.4. For $\alpha>-1$ and $\beta>0$,

$$
P_{n}^{(\alpha, \beta-1)}(x)=Q_{n}^{(\alpha, \beta)}(x)+d_{n-1}(\lambda) Q_{n-1}^{(\alpha, \beta)}(x), \quad n \geq 1,
$$

and for $\alpha, \beta>-1$,

$$
\frac{n+\alpha+\beta}{2} P_{n-1}^{(\alpha+1, \beta)}(x)=\left(Q_{n}^{(\alpha, \beta)}(x)\right)^{\prime}+d_{n-1}(\lambda)\left(Q_{n-1}^{(\alpha, \beta)}(x)\right)^{\prime}, \quad n \geq 1
$$

Proof. The first statement follows from Proposition 3.1 and (2.4). The second one follows by taking derivatives in (3.10) and using (2.6).

Using (3.10) in a recursive way, the representation of the polynomials $Q_{n}^{(\alpha, \beta)}$ in terms of the elements of the sequence $\left\{P_{n}^{(\alpha, \beta-1)}(x)\right\}_{n=0}^{\infty}$ becomes

$$
Q_{n}^{(\alpha, \beta)}(x)=\sum_{k=0}^{n}(-1)^{k} b_{k}^{(n)}(\lambda) P_{n-k}^{(\alpha, \beta-1)}(x)
$$

where $b_{k}^{(n)}(\lambda)=\prod_{j=1}^{k} d_{n-j}(\lambda)$ and $b_{0}^{(n)}(\lambda)=1$.

Proposition 3.5. There exists a constant $c>1$ such that the coefficients $b_{k}^{(n)}(\lambda)$ in (3.11) satisfy $b_{k}^{(n)}(\lambda)<c\left(1 / n 2^{k}\right)$ for all $n \geq 1$ and $1 \leq k \leq n$.

Proof. From (3.9), we have $\lim _{n} 2(n+1) d_{n}(\lambda)=0$. Thus, there exist $n_{0} \in N$ and a constant $c>1$ such that $2(n+1) d_{n}(\lambda)<1$ for all $n \geq n_{0}$ and $2(n+1) d_{n}(\lambda)<c$ for $n=1, \ldots, n_{0}-1$. Therefore, for $1 \leq k \leq n-n_{0}$,

$$
b_{k}^{(n)}(\lambda)=\prod_{j=1}^{k} d_{n-j}(\lambda)<\frac{1}{n 2^{k}}
$$

and for $n-n_{0} \leq k \leq n$,

$$
\begin{aligned}
b_{k}^{(n)}(\lambda) & =\prod_{j=1}^{n-n_{0}} d_{n-j}(\lambda) \prod_{j=n-n_{0}+1}^{k} d_{n-j}(\lambda) \\
& \leq \frac{1}{n 2^{n-n_{0}}}\left(\frac{c}{2}\right)^{k-n+n_{0}}=c^{k-n+n_{0}} \frac{1}{n 2^{k}} \leq c^{n_{0}} \frac{1}{n 2^{k}} .
\end{aligned}
$$


Journal of Inequalities and Applications

Proposition 3.6. (a) For the polynomials $Q_{n}^{(\alpha, \beta)}$, one obtains

$$
\left|Q_{n}^{(\alpha, \beta)}(x)\right| \leq c n^{-1 / 2}(1-x)^{-\alpha / 2-1 / 4}(1+x)^{-\beta / 2+1 / 4},
$$

for $x \in(-1,1), \alpha \geq-1 / 2$, and $\beta \geq 1 / 2$.

(b) For the polynomials $Q_{n}^{\prime(\alpha, \beta)}$, one has the following estimate:

$$
\left|Q_{n}^{\prime(\alpha, \beta)}(x)\right| \leq c n^{1 / 2}(1-x)^{-\alpha / 2-3 / 4}(1+x)^{-\beta / 2-1 / 4},
$$

$$
\text { for } x \in(-1,1), \alpha>-1 \text {, and } \beta \geq-1 / 2 \text {. }
$$

Proof. (a) Using (3.12), we have the following:

$$
\left|Q_{n}^{(\alpha, \beta)}(\cos \theta)\right| \leq \sum_{k=0}^{n} b_{k}^{(n)}(\lambda)\left|P_{n-k}^{(\alpha, \beta-1)}(\cos \theta)\right|
$$

From (2.7), it is straightforward to prove that, for $\alpha, \beta \geq-1 / 2$ and $k=0,1, \ldots, n-1$,

$$
\left|P_{n-k}^{(\alpha, \beta)}(\cos \theta)\right| \leq c \sqrt{\frac{n}{n-k}} n^{-1 / 2} \theta^{-\alpha-1 / 2}(\pi-\theta)^{-\beta-1 / 2} .
$$

Thus, according to Proposition 3.5,

$$
\begin{aligned}
\left|Q_{n}^{(\alpha, \beta)}(\cos \theta)\right| & \leq \sum_{k=0}^{n} b_{k}^{(n)}(\lambda)\left|P_{n-k}^{(\alpha, \beta-1)}(\cos \theta)\right| \\
& \leq c b_{n}^{(n)}(\lambda)+c n^{-1 / 2} \theta^{-\alpha-1 / 2}(\pi-\theta)^{-\beta+1 / 2} \sum_{k=0}^{n-1} \frac{1}{2^{k}} \\
& \leq c n^{-1 / 2} \theta^{-\alpha-1 / 2}(\pi-\theta)^{-\beta+1 / 2} .
\end{aligned}
$$

On the other hand, from (3.11), the proof of the case (b) can be done in a similar way.

Proposition 3.7. Let $\alpha, \beta>-1$, then

$$
\begin{gathered}
\left|Q_{n}^{(\alpha, \beta)}(x)\right| \leq \begin{cases}c n^{\alpha}, & \text { for } x \in[0,1], \alpha \geq-\frac{1}{2}, \\
c n^{\beta-1}, & \text { for } x \in[-1,0], \beta \geq \frac{1}{2} \\
c n^{-1 / 2}, & \text { for } x \in[-1,1], \alpha \leq-\frac{1}{2}, \beta \leq \frac{1}{2},\end{cases} \\
\left|Q_{n}^{\prime(\alpha, \beta)}(x)\right| \leq \begin{cases}c n^{\alpha+1}, & \text { for } x \in[0,1], \alpha>-1, \\
c n^{\beta+1}, & \text { for } x \in[-1,0], \beta \geq-\frac{1}{2} .\end{cases}
\end{gathered}
$$


Proof. Taking into account that the Jacobi polynomials satisfy the following (see [12, paragraph below Theorem 7.32.1]):

$$
\left|P_{n}^{(\alpha, \beta)}(x)\right| \leq \begin{cases}c n^{\alpha}, & \text { for } x \in[0,1], \alpha \geq-\frac{1}{2} \\ c n^{\beta}, & \text { for } x \in[-1,0], \beta \geq-\frac{1}{2} \\ c n^{-1 / 2}, & \text { for } x \in[-1,1], \alpha \leq-\frac{1}{2}, \beta \leq-\frac{1}{2}\end{cases}
$$

for $n \geq 1$, thus, for $0 \leq j \leq n-1$,

$$
\left|P_{n-j}^{(\alpha, \beta)}(x)\right| \leq \begin{cases}c\left(\frac{n-j}{n}\right)^{\alpha} n^{\alpha}, & \text { for } x \in[0,1], \alpha \geq-\frac{1}{2} \\ c\left(\frac{n-j}{n}\right)^{\beta} n^{\beta}, & \text { for } x \in[-1,0], \beta \geq-\frac{1}{2} \\ c\left(\frac{n-j}{n}\right)^{-1 / 2} n^{-1 / 2}, & \text { for } x \in[-1,1], \alpha \leq-\frac{1}{2}, \beta \leq-\frac{1}{2}\end{cases}
$$

As a consequence, the statement follows from the latter estimates and arguments similar to those we used in the proof of Proposition 3.6.

Corollary 3.8. For $\alpha \geq-1 / 2$ and $\beta \geq 1 / 2$,

$$
\left|Q_{n}^{(\alpha, \beta)}(\cos \theta)\right| \leq c A(n, \alpha, \beta-1, \theta)
$$

and for $\alpha>-1$ and $\beta \geq-1 / 2$,

$$
\left|Q_{n}^{\prime(\alpha, \beta)}(\cos \theta)\right| \leq c A(n, \alpha+1, \beta, \theta),
$$

where

$$
A(n, \alpha, \beta, \theta)= \begin{cases}n^{-1 / 2}\left(\theta^{-\alpha-1 / 2}(\pi-\theta)^{-\beta-1 / 2}\right), & \text { if } \frac{c}{n} \leq \theta \leq \pi-\frac{c}{n} \\ n^{\alpha}, & \text { if } 0 \leq \theta \leq \frac{c}{n} \\ n^{\beta}, & \text { if } \pi-\frac{c}{n} \leq \theta \leq \pi .\end{cases}
$$

Proof. The inequality

$$
n^{\alpha} \leq c n^{-1 / 2} \theta^{-\alpha-1 / 2}
$$


holds for $\theta \in(0, c / n]$, as well as

$$
n^{\beta} \leq c n^{-1 / 2}(\pi-\theta)^{-\beta-1 / 2}
$$

holds for $\theta \in[\pi-c / n, \pi)$. Therefore, the statement follows from Propositions 3.6 and 3.7.

Next, we show that the Jacobi-Sobolev polynomial $Q_{n}^{(\alpha, \beta)}(x)$ attains its maximum in $[-1,1]$ at the end points. To be more precise, consider the following.

Proposition 3.9. (a) For $\alpha \geq-1 / 2, \beta \geq 1 / 2$, and $q=\max \{\alpha, \beta-1\}$,

$$
\max _{-1 \leq x \leq 1}\left|Q_{n}^{(\alpha, \beta)}(x)\right|=\left|Q_{n}^{(\alpha, \beta)}(a)\right| \sim n^{q},
$$

where $a=1$ if $q=\alpha$, and $a=-1$ if $q=\beta-1$.

(b) For $\alpha>-1, \beta \geq-1 / 2$, and $q=\max \{\alpha+1, \beta\}$,

$$
\max _{-1 \leq x \leq 1}\left|Q_{n}^{\prime(\alpha, \beta)}(x)\right|=\left|Q_{n}^{\prime(\alpha, \beta)}(b)\right| \sim n^{q+1},
$$

where $b=1$ if $q=\alpha+1$, and $b=-1$ if $q=\beta$.

Proof. (a) We will prove only the case $q=\alpha$. If $q=\beta-1$, the the proof can be done in a similar way. From (3.9), (3.10), and Proposition 3.7,

$$
Q_{n}^{(\alpha, \beta)}(x)=P_{n}^{(\alpha, \beta-1)}(x)-d_{n-1}(\lambda) Q_{n-1}^{(\alpha, \beta)}(x)=P_{n}^{(\alpha, \beta-1)}(x)-O\left(n^{\alpha-2}\right) .
$$

Now, from [12, Theorem 7.32.1] and Proposition 3.7, the result follows.

Taking into account (2.6), the case (b) can be proved in a similar way.

Next, we deduce a Mehler-Heine type formula for $Q_{n}^{(\alpha, \beta)}$ and $\left(Q_{n}^{(\alpha, \beta)}\right)^{\prime}$.

Proposition 3.10. Let $\alpha, \beta>-1$. Uniformly on compact subsets of $C$, one gets

(a)

$$
\lim _{n \rightarrow \infty} n^{-\alpha} Q_{n}^{(\alpha, \beta)}\left(\cos \frac{z}{n}\right)=\left(\frac{z}{2}\right)^{-\alpha} J_{\alpha}(z)
$$

(b)

$$
\lim _{n \rightarrow \infty} n^{-\alpha-2} Q_{n}^{\prime(\alpha, \beta)}\left(\cos \frac{z}{n}\right)=\left(\frac{z}{2}\right)^{-(\alpha+1)} J_{\alpha+1}(z)
$$

Proof. (a) Multiplying in (3.8) by $(n+1)^{-\alpha}$, we obtain

$$
V_{n}(z)=Y_{n}(z)+D_{n-1}(\lambda) Y_{n-1}(z),
$$


where $V_{n}(z)=(n+1)^{-\alpha}\left[(n+\alpha+\beta) /(2 n+\alpha+\beta) P_{n}^{(\alpha, \beta)}(\cos (z / n))+((n+\alpha) /(2 n+\alpha+\beta)) P_{n-1}^{(\alpha, \beta)}\right.$ $\times(\cos (z / n))], Y_{n}(z)=(n+1)^{-\alpha} Q_{n}^{(\alpha, \beta)}(\cos (z / n))$ and $D_{n-1}(\lambda)=d_{n-1}(\lambda)(n /(n+1))^{\alpha} \cong c / n^{2}$ according to (3.9).

Using the above relation in a recursive way, we obtain

$$
Y_{n}(z)=\sum_{k=0}^{n}(-1)^{k} B_{k}^{(n)}(\lambda) V_{n-k}(z)
$$

where $B_{k}^{(n)}(\lambda)=\prod_{j=1}^{k} D_{n-j}(\lambda)$ and $B_{0}^{(n)}(\lambda)=1$. Moreover, by using the same argument as in Proposition 3.5, we have $B_{k}^{(n)}(\lambda)<c\left(1 / n 2^{k}\right)$ for every $n \geq 1$ and $1 \leq k \leq n$. Thus,

$$
\left|Y_{n}(z)\right| \leq \sum_{k=0}^{n} B_{k}^{(n)}(\lambda)\left|V_{n-k}(z)\right|
$$

On the other hand, from (2.8), we have that $\left\{V_{n}(z)\right\}_{n=0}^{\infty}$ is uniformly bounded on compact subsets of $\mathbf{C}$. Thus, for a fixed compact set $K \subset \mathbf{C}$, there exists a constant $C$, depending only on $K$, such that when $z \in K$,

$$
\left|V_{n}(z)\right|<C, \quad n \geq 1
$$

Thus, the sequence $\left\{Y_{n}(z)\right\}_{n=0}^{\infty}$ is uniformly bounded on $K \subset \mathrm{C}$. As a conclusion,

$$
Y_{n}(z)=V_{n}(z)+O\left(n^{-2}\right), \quad z \in K,
$$

and using (2.8), we obtain the result.

(b) Since we have uniform convergence in (3.31), taking derivatives and using some properties of Bessel functions, we obtain (3.32).

Now, we give the inner strong asymptotics of $Q_{n}^{(\alpha, \beta)}$ on $(-1,1)$.

Proposition 3.11. Let $\theta \in[\epsilon, \pi-\epsilon]$ and $\epsilon>0$. For $\alpha \geq-1 / 2, \beta \geq 1 / 2$, one has

$$
Q_{n}^{(\alpha, \beta)}(\cos \theta)=\pi^{-1 / 2} n^{-1 / 2}\left[\left(\sin \frac{\theta}{2}\right)^{-\alpha-1 / 2}\left(\cos \frac{\theta}{2}\right)^{-\beta+1 / 2} \cos \left(k_{1} \theta+\gamma\right)+O\left(n^{-1}\right)\right],
$$

and for $\alpha>-1, \beta \geq-1 / 2$, one has

$$
\begin{aligned}
& Q_{n}^{\prime(\alpha, \beta)}(\cos \theta) \\
& \quad=\pi^{-1 / 2} \frac{(n+\alpha+\beta+1)(n-1)^{-1 / 2}}{2}\left[\left(\sin \frac{\theta}{2}\right)^{-\alpha-3 / 2}\left(\cos \frac{\theta}{2}\right)^{-\beta-1 / 2} \cos \left(k_{1} \theta+\gamma_{1}\right)+O\left(n^{-1}\right)\right],
\end{aligned}
$$

where $k_{1}=n+(\alpha+\beta) / 2, \gamma=-(\alpha+1 / 2) \pi / 2$, and $\gamma_{1}=-(\alpha+3 / 2) \pi / 2$. 
Proof. From Proposition 3.6(a), the sequence $\left\{n^{1 / 2} Q_{n}^{(\alpha, \beta)}(x)\right\}_{n=1}^{\infty}$ is uniformly bounded on compact subsets of $(-1,1)$. Multiplication by $n^{1 / 2}$ in (3.10) yields

$$
n^{1 / 2} Q_{n}^{(\alpha, \beta)}(x)=n^{1 / 2} P_{n}^{(\alpha, \beta-1)}(x)-d_{n-1}(\lambda) \sqrt{\frac{n}{n-1}}(n-1)^{1 / 2} Q_{n-1}^{(\alpha, \beta)}(x)
$$

Since

$$
d_{n-1}(\lambda) \sqrt{\frac{n}{n-1}}=O\left(\frac{1}{n^{2}}\right)
$$

we have

$$
n^{1 / 2} Q_{n}^{(\alpha, \beta)}(x)=n^{1 / 2} P_{n}^{(\alpha, \beta-1)}(x)+O\left(n^{-2}\right) .
$$

Now, (3.38) follows from (2.9).

Concerning (3.39), it can be obtained in a similar way by using (3.11) and Proposition 3.6(b).

Next, we obtain an estimate for the Sobolev norms of the Jacobi-Sobolev polynomials.

Proposition 3.12. For $\alpha>-1 / 2, \alpha+1 \geq \beta \geq-1 / 2$, and $1 \leq p \leq \infty$, one has

$$
\left\|Q_{n}^{(\alpha, \beta)}\right\|_{S_{p}^{\alpha, \beta}} \sim \begin{cases}n^{1 / 2}, & \text { if } \frac{4(\alpha+2)}{2 \alpha+3}>p, \\ n^{1 / 2} \log n, & \text { if } \frac{4(\alpha+2)}{2 \alpha+3}=p, \\ n^{\alpha+2-(2 \alpha+4) / p}, & \text { if } \frac{4(\alpha+2)}{2 \alpha+3}<p .\end{cases}
$$

Notice that if $p=\infty$, then we have Proposition 3.9(b). Thus, in the proof we will assume $1 \leq p<\infty$.

Proof. In order to establish the upper bound in (3.38), it is enough to prove that

$$
\left\|Q_{n}^{(\alpha, \beta)}\right\|_{S_{p}^{\alpha, \beta}} \leq c n\left\|P_{n}^{(\alpha+1, \beta)}\right\|_{L^{p}\left(d \mu_{\alpha+1, \beta}\right)}
$$

Using (3.8) in a recurrence way and then Minkowski's inequality, we obtain

$$
\left\|Q_{n}^{(\alpha, \beta)}\right\|_{L^{p}\left(d \mu_{\alpha, \beta}\right)} \leq c \sum_{k=0}^{n} b_{k}^{(n)}(\lambda)\left\|P_{n-k}^{(\alpha, \beta)}\right\|_{L^{p}\left(d \mu_{\alpha, \beta}\right)}+c \sum_{k=0}^{n} b_{k}^{(n)}(\lambda)\left\|P_{n-k-1}^{(\alpha, \beta)}\right\|_{L^{p}\left(d \mu_{\alpha, \beta}\right)}
$$


On the other hand, for $\alpha, \beta>-1$ and $k=0,1, \ldots, n,(2.10)$ implies

$$
(n-k)^{1 / 2}\left\|P_{n-k}^{(\alpha, \beta)}\right\|_{L^{p}\left(d \mu_{\alpha, \beta}\right)} \leq c n^{1 / 2}\left\|P_{n}^{(\alpha, \beta)}\right\|_{L^{p}\left(d \mu_{\alpha, \beta}\right)} .
$$

Thus,

$$
\left\|P_{n-k}^{(\alpha, \beta)}\right\|_{L^{p}\left(d \mu_{\alpha, \beta}\right)} \leq \sqrt{\frac{n}{n-k}}\left\|P_{n}^{(\alpha, \beta)}\right\|_{L^{p}\left(d \mu_{\alpha, \beta}\right)^{\prime}} \quad 0 \leq k \leq n-1 .
$$

On the other hand, from Proposition 3.5,

$$
\begin{aligned}
\sum_{k=0}^{n} b_{k}^{(n)}(\lambda)\left\|P_{n-k}^{(\alpha, \beta)}\right\|_{L^{p}\left(d \mu_{\alpha, \beta}\right)} & \leq c b_{n}^{(n)}(\lambda)+\sum_{k=0}^{n-1} b_{k}^{(n)}(\lambda)\left\|P_{n-k}^{(\alpha, \beta)}\right\|_{L^{p}\left(d \mu_{\alpha, \beta}\right)} \\
& \leq c\left\|P_{n-1}^{(\alpha, \beta)}\right\|_{L^{p}\left(d \mu_{\alpha, \beta}\right)} \sum_{i=0}^{n-1} \frac{1}{2^{k}} \leq c\left\|P_{n}^{(\alpha, \beta)}\right\|_{L^{p}\left(d \mu_{\alpha, \beta}\right)} .
\end{aligned}
$$

Thus,

$$
\left\|Q_{n}^{(\alpha, \beta)}\right\|_{L^{p}\left(d \mu_{\alpha, \beta}\right)} \leq c\left\|P_{n}^{(\alpha, \beta)}\right\|_{L^{p}\left(d \mu_{\alpha, \beta}\right)} \leq c n\left\|P_{n}^{(\alpha+1, \beta)}\right\|_{L^{p}\left(d \mu_{\alpha+1, \beta}\right)} .
$$

In the same way as above, we conclude that

$$
\left\|Q_{n}^{\prime(\alpha, \beta)}\right\|_{L^{p}\left(d \mu_{\alpha+1, \beta)}\right.} \leq c n \sum_{k=0}^{n} b_{k}^{(n)}(\lambda)\left\|P_{n-k-1}^{(\alpha+1, \beta)}\right\|_{L^{p}\left(d \mu_{\alpha+1, \beta}\right)} \leq c n\left\|P_{n}^{(\alpha+1, \beta)}\right\|_{L^{p}\left(d \mu_{\alpha+1, \beta}\right)} .
$$

Thus, (3.44) follows from (3.49) and (3.50).

In order to prove the lower bound in relation (3.43), we will need the following.

Proposition 3.13. For $\alpha>-1$ and $1 \leq p<\infty$, one has

$$
\left\|Q_{n}^{\prime(\alpha, \beta)}\right\|_{L^{p}\left(d \mu_{\alpha+1, \beta)} \geq c\right.} \geq \begin{array}{ll}
n^{1 / 2}, & \text { if } \frac{4(\alpha+2)}{2 \alpha+3}>p, \\
n^{1 / 2} \log n, & \text { if } \frac{4(\alpha+2)}{2 \alpha+3}=p, \\
n^{\alpha+2-(2 \alpha+4) / p}, & \text { if } \frac{4(\alpha+2)}{2 \alpha+3}<p .
\end{array}
$$


Proof. We will use a technique similar to [12, Theorem 7.34]. According to (3.11),

$$
\begin{aligned}
\int_{0}^{\pi / 2} \theta^{2 \alpha+3}\left|Q_{n}^{\prime(\alpha, \beta)}(\cos \theta)\right|^{p} d \theta & >\int_{0}^{\omega / n} \theta^{2 \alpha+3}\left|Q_{n}^{\prime(\alpha, \beta)}(\cos \theta)\right|^{p} d \theta \\
& \geq c n^{-2 \alpha-4} \int_{0}^{\omega} t^{2 \alpha+3}\left|Q_{n}^{\prime(\alpha, \beta)}\left(\cos \frac{t}{n}\right)\right|^{p} d t \\
& \cong c n^{p(\alpha+2)-2 \alpha-4} \int_{0}^{\omega} t^{2 \alpha+3}\left|t^{-(\alpha+1)} J_{\alpha+1}(t)\right|^{p} d t \\
& =c n^{p(\alpha+2)-2 \alpha-4} \int_{0}^{\omega} t^{2 \alpha+3-p(\alpha+1)}\left|J_{\alpha+1}(t)\right|^{p} d t .
\end{aligned}
$$

On the other hand, Stempak's lemma (see [16, Lemma 2.1]), for $\gamma>-1-p \alpha$ and $1 \leq p<\infty$, implies

$$
\int_{0}^{\omega} t^{\gamma}\left|J_{\alpha+1}(t)\right|^{p} d t \sim \begin{cases}c, & \text { if } \gamma<\frac{p}{2}-1 \\ c \log \omega, & \text { if } \gamma=\frac{p}{2}-1 .\end{cases}
$$

Thus, for $4(\alpha+2) /(2 \alpha+3) \leq p$ and $\omega$ large enough, (3.51) follows.

Finally, from (3.39) we obtain the following:

$$
\int_{0}^{\pi / 2} \theta^{2 \alpha+3}\left|Q_{n}^{\prime(\alpha, \beta)}(\cos \theta)\right|^{p} d \theta>\int_{\pi / 4}^{\pi / 2} \theta^{2 \alpha+3}\left|Q_{n}^{\prime(\alpha, \beta)}(\cos \theta)\right|^{p} d \theta \sim n^{p / 2}
$$

For the proof of Proposition 3.12, from (3.51), for $\alpha>-1$ and $1 \leq p<\infty$, we get

$$
\left\|Q_{n}^{(\alpha, \beta)}\right\|_{S_{p}^{\alpha, \beta}} \geq c \begin{cases}n^{1 / 2}, & \text { if } \frac{4(\alpha+2)}{2 \alpha+3}>p, \\ n^{1 / 2} \log n, & \text { if } \frac{4(\alpha+2)}{2 \alpha+3}=p, \\ n^{\alpha+2-(2 \alpha+4) / p,}, & \text { if } \frac{4(\alpha+2)}{2 \alpha+3}<p .\end{cases}
$$

Thus, using (3.44) and (3.55), the statement follows.

\section{A Cohen Type Inequality for Jacobi-Sobolev Expansions}

For $f \in S_{1}^{\alpha, \beta}$, its Fourier expansion in terms of Jacobi-Sobolev polynomials is

$$
\sum_{k=0}^{\infty} \widehat{f}(k) Q_{k}^{(\alpha, \beta)}(x),
$$


where

$$
\widehat{f}(k)=\left(\left\|Q_{k}^{(\alpha, \beta)}\right\|_{S_{2}^{\alpha, \beta}}^{2}\right)^{-1}\left\langle f, Q_{k}^{(\alpha, \beta)}\right\rangle, \quad k=0,1, \ldots
$$
77]),

The Cesàro means of order $\delta$ of the expansion (4.1) is defined by (see $[17$, pages 76 -

$$
\sigma_{n}^{\delta} f(x)=\sum_{k=0}^{n} \frac{C_{n-k}^{\delta}}{C_{n}^{\delta}} \widehat{f}(k) Q_{k}^{(\alpha, \beta)}(x),
$$

where $C_{k}^{\delta}=\left(\begin{array}{c}k+\delta \\ k\end{array}\right)$.

For a function $f \in S_{p}^{\alpha, \beta}$ and a fixed sequence $\left\{c_{k, n}\right\}_{k=0}^{n}, n \in \mathbf{N} \cup\{\mathbf{0}\}$, of real numbers with $c_{n-1, n}=o\left(n^{2} c_{n, n}\right)$, we define the operators $T_{n}^{\alpha, \beta}$ by

$$
T_{n}^{\alpha, \beta}(f)=\sum_{k=0}^{n} c_{k, n} \widehat{f}(k) Q_{k}^{(\alpha, \beta)}
$$
result.

Let $q_{0}=(4 \alpha+8) /(2 \alpha+3)$ and let $p_{0}$ be the conjugate of $q_{0}$. Now, we can state our main

Theorem 4.1. For $\alpha>-1 / 2$ and $\alpha+1 \geq \beta \geq-1 / 2$, one has

$$
\left\|T_{n}^{\alpha, \beta}\right\|_{\left[S_{p}^{\alpha}\right]} \geq c\left|c_{n, n}\right| \begin{cases}n^{(2 \alpha+4) / p-(2 \alpha+5) / 2}, & \text { if } 1 \leq p<p_{0} \\ (\log n)^{(2 \alpha+3) /(4 \alpha+8)}, & \text { if } p=p_{0}, p=q_{0} \\ n^{(2 \alpha+3) / 2-(2 \alpha+4) / p}, & \text { if } q_{0}<p \leq \infty .\end{cases}
$$

Corollary 4.2. Let $\alpha, \beta, p_{0}, q_{0}$, and $p$ be as in Theorem 4.1. For $c_{k, n}=1, k=0, \ldots, n$, and for $p$ outside the interval $\left(p_{0}, q_{0}\right)$, one has

$$
\left\|\sigma_{n}^{0}\right\|_{\left[S_{p}^{\alpha, \beta}\right]} \longrightarrow \infty, \quad n \longrightarrow \infty
$$

For $c_{k, n}=C_{n-k}^{\delta} / C_{n}^{\delta}, 0 \leq k \leq n$, Theorem 4.1 yields the following.

Corollary 4.3. For $\alpha>-1 / 2$ and $\alpha+1 \geq \beta \geq-1 / 2$, one has

$$
\begin{aligned}
& 0<\delta<\frac{2 \alpha+4}{p}-\frac{2 \alpha+5}{2}, \quad \text { if } 1 \leq p<p_{0}, \\
& 0<\delta<\frac{2 \alpha+3}{2}-\frac{2 \alpha+4}{p}, \quad \text { if } q_{0}<p \leq \infty
\end{aligned}
$$


and $p \notin\left[p_{0}, q_{0}\right]$,

$$
\left\|\sigma_{n}^{\delta}\right\|_{\left[S_{p}^{\alpha, \beta}\right]} \longrightarrow \infty, \quad n \longrightarrow \infty
$$

We will use the following as test functions (see [10, formula (2.8)], and [11, formula (22.7.19)]):

$$
\begin{aligned}
g_{n}^{\alpha, \beta-1, j}(x) & =\left(1-x^{2}\right)^{j} P_{n}^{(\alpha+j, \beta-1+j)}(x)=\sum_{m=0}^{2 j} c_{m, j}(\alpha, \beta-1, n) P_{n+m}^{(\alpha, \beta-1)}(x) \\
& =\sum_{m=0}^{2 j} c_{m, j}(\alpha, \beta-1, n)\left(A_{n+m}(\alpha, \beta) P_{n+m}^{(\alpha, \beta)}(x)+B_{n+m}(\alpha, \beta) P_{n+m-1}^{(\alpha, \beta)}(x)\right)
\end{aligned}
$$

where $j \in \mathbf{N} \backslash\{\mathbf{1}\}$, and

$$
\begin{aligned}
& c_{0, j}(\alpha, \beta, n)= \frac{4^{j} \Gamma(n+\alpha+j+1) \Gamma(n+\beta+j+1) \Gamma(2 n+\alpha+\beta+2)}{\Gamma(n+\alpha+1) \Gamma(n+\beta+1) \Gamma(2 n+\alpha+\beta+2 j+2)}, \\
& c_{1, j}(\alpha, \beta, n)=-\frac{4^{j} A_{1}^{-j-1}(n+1) \Gamma(n+\alpha+j+1) \Gamma(n+\beta+j+1) \Gamma(2 n+\alpha+\beta+3)}{(2 n+\alpha+\beta+j+2) \Gamma(n+\alpha+1) \Gamma(n+\beta+2) \Gamma(2 n+\alpha+\beta+2 j+2)} \\
&+\frac{4^{j} A_{1}^{-j-1}(n+1) \Gamma(n+\alpha+j+1) \Gamma(n+\beta+j+2) \Gamma(2 n+\alpha+\beta+4)}{(2 n+\alpha+\beta+j+3) \Gamma(n+\alpha+2) \Gamma(n+\beta+2) \Gamma(2 n+\alpha+\beta+2 j+3)}, \\
& c_{2 j, j}(\alpha, \beta, n)= \frac{(-4)^{j} \Gamma(n+2 j+1) \Gamma(2 n+2 j+\alpha+\beta+1)}{\Gamma(n+1) \Gamma(2 n+4 j+\alpha+\beta+1)}, \\
& A_{n}(\alpha, \beta)=\frac{n+\alpha+\beta}{2 n+\alpha+\beta}, \quad B_{n}(\alpha, \beta)=\frac{n+\alpha}{2 n+\alpha+\beta} .
\end{aligned}
$$

Applying the operator $T_{n}^{\alpha, \beta}$ to $g_{n}^{\alpha, \beta-1, j}$, for some $j>\alpha+5 / 2-2(\alpha+2) / p$, we get

$$
T_{n}^{\alpha, \beta}\left(g_{n}^{\alpha, \beta-1, j}\right)=\sum_{k=0}^{n} c_{k, n}\left(g_{n}^{\alpha, \beta-1, j}\right) \sim(k) Q_{k}^{(\alpha, \beta)}
$$

where

$$
\left(g_{n}^{\alpha, \beta-1, j}\right) \sim(k)=\left(\left\|Q_{k}^{(\alpha, \beta)}\right\|_{S_{2}^{\alpha, \beta}}^{2}\right)^{-1}\left\langle g_{n}^{\alpha, \beta-1, j}, Q_{k}^{(\alpha, \beta)}\right\rangle, \quad k=0,1, \ldots, n,
$$

and using (2.3) and (3.3), we deduce

$$
\left\|Q_{n}^{(\alpha, \beta)}\right\|_{S_{2}^{\alpha, \beta}}^{2} \cong \lambda 2^{\alpha+\beta} n
$$


Taking into account (4.9), for $0 \leq k \leq n-2$,

$$
\int_{-1}^{1} g_{n}^{\alpha, \beta-1, j}(x) Q_{k}^{(\alpha, \beta)}(x) d \mu_{\alpha, \beta}(x)=0 .
$$

If $k=n-1$, then we get

$$
\begin{aligned}
\int_{-1}^{1} g_{n}^{\alpha, \beta-1, j}(x) Q_{n-1}^{(\alpha, \beta)}(x) d \mu_{\alpha, \beta}(x)= & c_{0, j}(\alpha, \beta, n) A_{n-1}(\alpha, \beta) B_{n}(\alpha, \beta) \\
& \times \int_{-1}^{1} P_{n-1}^{(\alpha, \beta)}(x) P_{n-1}^{(\alpha, \beta)}(x) d \mu_{\alpha, \beta}(x) \cong 2^{\alpha+\beta+2 j-2} n^{-1} .
\end{aligned}
$$

If $k=n$, then

$$
\begin{aligned}
\int_{-1}^{1} g_{n}^{\alpha, \beta-1, j}(x) Q_{n}^{(\alpha, \beta)}(x) d \mu_{\alpha, \beta}(x) \\
=c_{0, j}(\alpha, \beta, n)\left(A_{n}(\alpha, \beta)\right)^{2} \int_{-1}^{1} P_{n}^{(\alpha, \beta)}(x) P_{n}^{(\alpha, \beta)}(x) d \mu_{\alpha, \beta}(x) \\
\quad+c_{0, j}(\alpha, \beta, n)\left(B_{n}(\alpha, \beta)\right)^{2} \int_{-1}^{1} P_{n-1}^{(\alpha, \beta)}(x) P_{n-1}^{(\alpha, \beta)}(x) d \mu_{\alpha, \beta}(x) \\
\quad-c_{0, j}(\alpha, \beta, n) A_{n-1}(\alpha, \beta) B_{n}(\alpha, \beta) b_{1}^{(n)}(\lambda) \int_{-1}^{1} P_{n-1}^{(\alpha, \beta)}(x) P_{n-1}^{(\alpha, \beta)}(x) d \mu_{\alpha, \beta}(x) \\
\quad+c_{1, j}(\alpha, \beta, n) A_{n}(\alpha, \beta) B_{n+1}(\alpha, \beta) \int_{-1}^{1} P_{n}^{(\alpha, \beta)}(x) P_{n}^{(\alpha, \beta)}(x) d \mu_{\alpha, \beta}(x) \cong 2^{\alpha+\beta+2 j-1} n^{-1} .
\end{aligned}
$$

On the other hand, for $0 \leq k \leq n-1$,

$$
\int_{-1}^{1}\left(g_{n}^{\alpha, \beta-1, j}(x)\right)^{\prime}\left(Q_{k}^{(\alpha, \beta)}(x)\right)^{\prime} d \mu_{\alpha+1, \beta}(x)=0,
$$

and for $k=n$,

$$
\begin{aligned}
& \int_{-1}^{1}\left(g_{n}^{\alpha, \beta-1, j}(x)\right)^{\prime}\left(Q_{n}^{(\alpha, \beta)}(x)\right)^{\prime} d \mu_{\alpha+1, \beta}(x) \\
& \quad=\left(\frac{n+\alpha+\beta}{2}\right)^{2} c_{0, j}(\alpha, \beta-1, n) \int_{-1}^{1} P_{n-1}^{(\alpha+1, \beta)}(x) P_{n-1}^{(\alpha+1, \beta)}(x) d \mu_{\alpha+1, \beta}(x) \\
& \quad \cong 2^{\alpha+\beta+2 j-1} n .
\end{aligned}
$$


Thus,

$$
\begin{gathered}
\left\langle g_{n}^{\alpha, \beta-1, j}, Q_{k}^{(\alpha, \beta)}\right\rangle=0, \quad \text { if } 0 \leq k \leq n-2, \\
\left\langle g_{n}^{\alpha, \beta-1, j}, Q_{n-1}^{(\alpha, \beta)}\right\rangle \cong 2^{\alpha+\beta+2 j-2} n^{-1}, \\
\left\langle g_{n}^{\alpha, \beta-1, j}, Q_{n}^{(\alpha, \beta)}\right\rangle \cong 2^{\alpha+\beta+2 j-1} n .
\end{gathered}
$$

As a conclusion,

$$
\begin{gathered}
\left(g_{n}^{\alpha, \beta-1, j}\right) \sim(k)=0, \quad \text { if } 0 \leq k \leq n-2, \\
\left(g_{n}^{\alpha, \beta-1, j}\right) \sim(n-1) \cong \frac{2^{2 j-2}}{\lambda n^{2}}, \\
\left(g_{n}^{\alpha, \beta-1, j}\right) \smile(n) \cong \frac{2^{2 j-2}}{\lambda} .
\end{gathered}
$$

Now, we will estimate

$$
\left\|g_{n}^{\alpha, \beta-1, j}\right\|_{S_{p}^{\alpha, \beta}}^{p}=\left\|g_{n}^{\alpha, \beta-1, j}\right\|_{L^{p}\left(d \mu_{\alpha, \beta}\right)}^{p}+\lambda\left\|\left(g_{n}^{\alpha, \beta-1, j}\right)^{\prime}\right\|_{L^{p}\left(d \mu_{\alpha+1, \beta}\right)}^{p} .
$$

From [10, formula (3.1)],

$$
\left\|g_{n}^{\alpha, \beta-1, j}\right\|_{L^{p}\left(d \mu_{\alpha, \beta}\right)}^{p} \leq c n^{-p / 2}
$$

for $j>\alpha+1 / 2-(2 \alpha+2) / p \geq \beta-1 / 2-(2 \beta+2) / p$.

On the other hand, from (2.6), (4.9), and [12, formula (4.5.4)], one has

$$
\begin{aligned}
\left(g_{n}^{\alpha, \beta-1, j}(x)\right)^{\prime}= & \left(\left(1-x^{2}\right)^{j} P_{n}^{(\alpha+j, \beta-1+j)}(x)\right)^{\prime} \\
= & -2 j\left(1-x^{2}\right)^{j-1} x P_{n}^{(\alpha+j, \beta-1+j)}(x)+\frac{n+\alpha+\beta+2 j}{2}\left(1-x^{2}\right)^{j} P_{n}^{(\alpha+1+j, \beta+j)}(x) \\
= & \frac{4 j(n+\alpha+j)}{2 n+\alpha+\beta+2 j}\left(1-x^{2}\right)^{j-1} P_{n}^{(\alpha-1+j, \beta-1+j)}(x) \\
& -\frac{4 j(n+1)}{2 n+\alpha+\beta+2 j}\left(1-x^{2}\right)^{j-1} P_{n+1}^{(\alpha-1+j, \beta-1+j)}(x) \\
& -2 j\left(1-x^{2}\right)^{j-1} P_{n}^{(\alpha+j, \beta-1+j)}(x)+\frac{n+\alpha+\beta+2 j}{2}\left(1-x^{2}\right)^{j} P_{n}^{(\alpha+1+j, \beta+j)}(x) .
\end{aligned}
$$


From (2.10), for $j>\max \{\alpha+3 / 2-(2 \alpha+4) / p, \beta+3 / 2-(2 \beta+2) / p\}$,

$$
\left\|\left(1-x^{2}\right)^{j-1} P_{n}^{(\alpha-1+j, \beta-1+j)}\right\|_{L^{p}\left(d \mu_{\alpha+1, \beta}\right)} \sim n^{-1 / 2},
$$

for $\alpha+1 \geq \beta$ and $j>\alpha+5 / 2-(2 \alpha+4) / p$,

$$
\left\|\left(1-x^{2}\right)^{j-1} P_{n}^{(\alpha+j, \beta-1+j)}\right\|_{L^{p}\left(d \mu_{\alpha+1, \beta}\right)} \sim n^{-1 / 2},
$$

and for $\alpha+1 \geq \beta$ and $j>\alpha+3 / 2-(2 \alpha+4) / p$,

$$
\left\|\left(1-x^{2}\right)^{j} P_{n}^{(\alpha+1+j, \beta+j)}\right\|_{L^{p}\left(d \mu_{\alpha+1, \beta}\right)} \sim n^{-1 / 2} .
$$

Thus, for $\alpha+1 \geq \beta$ and $j>\alpha+5 / 2-(2 \alpha+4) / p$,

$$
\left\|\left(g^{\alpha, \beta-1, j}\right)^{\prime}\right\|_{L^{p}\left(d \mu_{\alpha+1, \beta}\right)} \leq c n^{1 / 2}
$$

By using (4.22) and (4.27), we find from (4.21) that

$$
\left\|g_{n}^{\alpha, \beta-1, j}\right\|_{S_{p}^{\alpha, \beta}} \leq c n^{1 / 2}
$$

for $\alpha+1 \geq \beta$ and $j>\alpha+5 / 2-(2 \alpha+4) / p$.

Now, we can prove our main result.

Proof of Theorem 4.1. By duality, it is enough to assume that $q_{0} \leq p \leq \infty$. From (4.11), (4.20), and (4.28), one has

$$
\begin{aligned}
\left\|T_{n}^{\alpha, \beta}\right\|_{S_{p}^{\alpha, \beta}} \geq & {\left[\left\|g_{n}^{(\alpha, \beta-1, j)}\right\|_{S_{p}^{\alpha, \beta}}\right]^{-1}\left\|T_{n}^{\alpha, \beta}\left(g_{n}^{(\alpha, \beta-1, j)}\right)\right\|_{S_{p}^{\alpha, \beta}} } \\
\geq & c n^{-1 / 2}\left|c_{n, n}\left(g_{n}^{\alpha, \beta-1, j}\right) \sim(n)\right|\left\|Q_{n}^{(\alpha, \beta)}\right\|_{S_{p}^{\alpha, \beta}} \\
& -c n^{-1 / 2}\left|c_{n-1, n}\left(g_{n}^{\alpha, \beta-1, j}\right) \sim(n-1)\right|\left\|Q_{n-1}^{(\alpha, \beta)}\right\|_{S_{p}^{\alpha, \beta}} \\
\sim & c n^{-1 / 2}\left|c_{1} c_{n, n}\right|\left\|Q_{n}^{(\alpha, \beta)}\right\|_{S_{p}^{\alpha, \beta}}\left(1-\left|\frac{c_{2} c_{n-1, n}}{c_{1} n^{2} c_{n, n}}\right|\right) .
\end{aligned}
$$

Now from Proposition 3.12, the statement of the theorem follows. 


\section{Necessary Conditions for the Norm Convergence}

The problem of the convergence in the norm of partial sums of the Fourier expansions in terms of Jacobi polynomials has been discussed by many authors. See, for instance, [18-20] and the references therein.

Let $q_{n}^{(\alpha, \beta)}$ be the Jacobi-Sobolev orthonormal polynomials, that is,

$$
q_{n}^{(\alpha, \beta)}(x)=\left(\left\|Q_{n}^{(\alpha, \beta)}\right\|_{S_{2}^{\alpha, \beta}}\right)^{-1} Q_{n}^{(\alpha, \beta)}(x)
$$
nomials is

For $f \in S_{1}^{\alpha, \beta}$, the Fourier expansion in terms of Jacobi-Sobolev orthonormal poly-

$$
\sum_{k=0}^{\infty} \widehat{f}(k) q_{k}^{(\alpha, \beta)}(x)
$$

where

$$
\widehat{f}(k)=\left\langle f, q_{k}^{(\alpha, \beta)}\right\rangle, \quad k=0,1, \ldots
$$

Let $S_{n} f$ be the $n$th partial sum of the expansion (5.2) as follows:

$$
S_{n}(f, x)=\sum_{k=0}^{n} \widehat{f}(k) q_{k}^{(\alpha, \beta)}(x) .
$$

Theorem 5.1. Let $\alpha>-1 / 2, \alpha+1 \geq \beta \geq-1 / 2$, and $1<p<\infty$. If there exists a constant $c>0$ such that

$$
\left\|S_{n} f\right\|_{S_{p}^{\alpha, \beta}} \leq c\|f\|_{S_{p}^{\alpha, \beta}}
$$

for every $f \in S_{p}^{\alpha, \beta}$, then $p \in\left(p_{0}, q_{0}\right)$.

Proof. For the proof, we apply the same argument as in [19]. Assume that (5.5) holds, then

$$
\left\|\left\langle f, q_{n}^{(\alpha, \beta)}\right\rangle q_{n}^{(\alpha, \beta)}(x)\right\|_{S_{p}^{\alpha, \beta}}=\left\|S_{n} f-S_{n-1} f\right\|_{S_{p}^{\alpha, \beta}} \leq 2 c\|f\|_{S_{p}^{\alpha, \beta}}
$$

Therefore,

$$
\left\|q_{n}^{(\alpha, \beta)}(x)\right\|_{S_{p}^{\alpha, \beta}}\left\|q_{n}^{(\alpha, \beta)}(x)\right\|_{S_{q}^{\alpha}}<\infty
$$

where $p$ is the conjugate of $q$. 
On the other hand, from (3.43) we obtain the Sobolev norms of Jacobi-Sobolev orthonormal polynomials as follows:

$$
\left\|q_{n}^{(\alpha, \beta)}\right\|_{S_{p}^{\alpha, \beta}} \sim \begin{cases}c, & \text { if } p<q_{0}, \\ \log n, & \text { if } \mathrm{p}=\mathrm{q}_{0}, \\ n^{\alpha+3 / 2-(2 \alpha+4) / p}, & \text { if } p>q_{0},\end{cases}
$$

for $\alpha>-1 / 2, \alpha+1 \geq \beta \geq-1 / 2$, and $1 \leq p \leq \infty$. Now, from (5.8) it follows that the inequality (5.7) holds if and only if $p \in\left(p_{0}, q_{0}\right)$.

The proof of Theorem 5.1 is complete.

\section{Divergence Almost Everywhere}

For $\lambda=0$ and $\alpha=\beta=0$, Pollard [21] showed that for each $p<4 / 3$ there exists a function $f \in L^{p}(d x)$ such that its Fourier expansion (4.27) diverges almost everywhere on [-1,1]. Later on, Meaney [22] extended the result to $p=4 / 3$. Furthermore, he proved that this is a special case of a divergence result for the Fourier expansion in terms of Jacobi polynomials. The failure of almost everywhere convergence of the Fourier expansions associated with systems of orthogonal polynomials on $[-1,1]$ and Bessel systems has been discussed in $[16,23]$.

If the sequence $\left\{S_{n}(f)\right\}_{n \geq 0}$ is uniformly bounded on a set, say $E$, of positive measure in $[-1,1]$, then

$$
\left\|\widehat{f}(n) q_{n}^{(\alpha, \beta)}(x)\right\|_{S_{\infty, E}^{\alpha, \beta}, E}<\mathcal{C}, \quad n \in \mathbf{N}, x \in E .
$$

Therefore,

$$
\left\|\widehat{f}(n) q_{n}^{\prime(\alpha, \beta)}(x)\right\|<c, \quad n \in \mathbf{N},
$$

almost everywhere on E. From Egorov's Theorem, it follows that there is a subset $E_{1} \subset E$ of positive measure such that

$$
\left\|\widehat{f}(n) q_{n}^{\prime(\alpha, \beta)}(x)\right\|<c,
$$

uniformly for $x \in E_{1}$. On the other hand, from (3.39)

$$
\left|\widehat{f}(n)\left(\cos \left(k_{1} \theta+r_{1}\right)+O\left(n^{-1}\right)\right)\right|<c
$$

uniformly for $\cos \theta \in E_{1}$. Using the Cantor-Lebesgue Theorem, as described in [24, Section 1.5], (see also [17, page 316]), we obtain

$$
|\widehat{f}(n)|<c .
$$


Theorem 6.1. Let $\alpha>-1 / 2$ and $\alpha+1 \geq \beta \geq-1 / 2$. There is an $f \in S_{p}^{\alpha, \beta}, 1 \leq p \leq p_{0}$, whose Fourier expansion (5.2) diverges almost everywhere on $[-1,1]$ in the norm of $S_{\infty}^{\alpha, \beta}$.

Proof. Consider the linear functionals

$$
T_{n}(f)=\widehat{f}(n)=\left\langle f, q_{n}^{(\alpha, \beta)}\right\rangle,
$$

on $S_{p}^{\alpha, \beta}, 1 \leq p \leq p_{0}$. By using [1, Theorem 3.8], we have

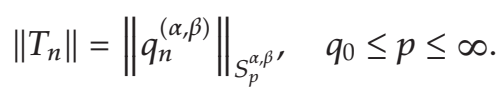

Thus, from (5.8),

$$
\sup _{n}\left\|T_{n}\right\|=\infty
$$

As a consequence of the Banach-Steinhaus theorem, there exists $f \in S_{p}^{\alpha, \beta}, 1 \leq p \leq p_{0}$, such that

$$
\sup _{n}\left|T_{n}(f)\right|=\infty
$$

Since this result contradicts (6.5), then for this $f$ the Fourier series diverges almost everywhere on $[-1,1]$ in the norm of $S_{\infty}^{\alpha, \beta}$.

\section{Acknowledgments}

The authors thank the careful revision of the paper by the referees. Their remarks and suggestions have contributed to improve the presentation. The work of the second author (F. Marcellán) has been supported by Direcci ón General de Investigación, Ministerio de Ciencia e Innovación of Spain, Grant no. MTM2009-12740-C03-01.

\section{References}

[1] R. A. Adams, Sobolev Spaces, vol. 6 of Pure and Applied Mathematics, Academic Press, New York, NY, USA, 1975.

[2] H. G. Meijer, "Determination of all coherent pairs," Journal of Approximation Theory, vol. 89, no. 3, pp. 321-343, 1997.

[3] D. H. Kim, K. H. Kwon, F. Marcellán, and G. J. Yoon, "Zeros of Jacobi-Sobolev orthogonal polynomials," International Mathematical Journal, vol. 4, no. 5, pp. 413-422, 2003.

[4] H. G. Meijer and M. G. de Bruin, "Zeros of Sobolev orthogonal polynomials following from coherent pairs," Journal of Computational and Applied Mathematics, vol. 139, no. 2, pp. 253-274, 2002.

[5] P. J. Cohen, “On a conjecture of Littlewood and idempotent measures," American Journal of Mathematics, vol. 82, pp. 191-212, 1960.

[6] B. Dreseler and P. M. Soardi, "A Cohen type inequality for ultraspherical series," Archiv der Mathematik, vol. 38, no. 3, pp. 243-247, 1982. 
[7] B. Dreseler and P. M. Soardi, "A Cohen-type inequality for Jacobi expansions and divergence of Fourier series on compact symmetric spaces," Journal of Approximation Theory, vol. 35, no. 3, pp. 214221, 1982.

[8] S. Giulini, P. M. Soardi, and G. Travaglini, "A Cohen type inequality for compact Lie groups," Proceedings of the American Mathematical Society, vol. 77, no. 3, pp. 359-364, 1979.

[9] G. H. Hardy and J. E. Littlewood, "A new proof of a theorem on rearrangements," Journal of the London Mathematical Society, vol. 23, pp. 163-168, 1948.

[10] C. Markett, "Cohen type inequalities for Jacobi, Laguerre and Hermite expansions," SIAM Journal on Mathematical Analysis, vol. 14, no. 4, pp. 819-833, 1983.

[11] M. Abramowitz and I. A. Stegun, Handbook of Mathematical Functions, Dover, New York, NY, USA, 1964.

[12] G. Szegő, Orthogonal Polynomials, vol. 23 of American Mathematical Society, Colloquium Publications, American Mathematical Society, Providence, RI, USA, 4th edition, 1975.

[13] P. Nevai, T. Erdélyi, and A. P. Magnus, "Generalized Jacobi weights, Christoffel functions, and Jacobi polynomials," SIAM Journal on Mathematical Analysis, vol. 25, no. 2, pp. 602-614, 1994.

[14] A. Iserles, P. E. Koch, S. P. Nørsett, and J. M. Sanz-Serna, "On polynomials orthogonal with respect to certain Sobolev inner products," Journal of Approximation Theory, vol. 65, no. 2, pp. 151-175, 1991.

[15] A. Martínez-Finkelshtein, J. J. Moreno-Balcázar, and H. Pijeira-Cabrera, "Strong asymptotics for Gegenbauer-Sobolev orthogonal polynomials," Journal of Computational and Applied Mathematics, vol. 81, no. 2, pp. 211-216, 1997.

[16] K. Stempak, “On convergence and divergence of Fourier-Bessel series," Electronic Transactions on Numerical Analysis, vol. 14, pp. 223-235, 2002.

[17] A. Zygmund, Trigonometric Series: Vols. I, II, Cambridge University Press, London, UK, 2nd edition, 1968.

[18] B. Muckenhoupt, "Mean convergence of Jacobi series," Proceedings of the American Mathematical Society, vol. 23, pp. 306-310, 1969.

[19] J. Newman and W. Rudin, "Mean convergence of orthogonal series," Proceedings of the American Mathematical Society, vol. 3, pp. 219-222, 1952.

[20] H. Pollard, "The mean convergence of orthogonal series. III," Duke Mathematical Journal, vol. 16, pp. 189-191, 1949.

[21] H. Pollard, "The convergence almost everywhere of Legendre series," Proceedings of the American Mathematical Society, vol. 35, pp. 442-444, 1972.

[22] C. Meaney, "Divergent Jacobi polynomial series," Proceedings of the American Mathematical Society, vol. 87, no. 3, pp. 459-462, 1983.

[23] J. J. Guadalupe, M. Pérez, F. J. Ruiz, and J. L. Varona, “Two notes on convergence and divergence a.e. of Fourier series with respect to some orthogonal systems," Proceedings of the American Mathematical Society, vol. 116, no. 2, pp. 457-464, 1992.

[24] Ch. Meaney, "Divergent Cesàro and Riesz means of Jacobi and Laguerre expansions," Proceedings of the American Mathematical Society, vol. 131, no. 10, pp. 3123-3218, 2003. 